\title{
Numerical Studies for Fractional Functional Differential Equations with Delay Based on BDF-Type Shifted Chebyshev Approximations
}

\author{
V. G. Pimenov and A. S. Hendy \\ Ural Federal University, Ulitsa Mira. 19. Yekaterinburg 620002, Russia \\ Correspondence should be addressed to A. S. Hendy; ahmed.hendy@fsc.bu.edu.eg \\ Received 6 November 2014; Accepted 8 March 2015 \\ Academic Editor: Gaston Mandata N'guérékata
}

Copyright (C) 2015 V. G. Pimenov and A. S. Hendy. This is an open access article distributed under the Creative Commons Attribution License, which permits unrestricted use, distribution, and reproduction in any medium, provided the original work is properly cited.

\begin{abstract}
Fractional functional differential equations with delay (FDDEs) have recently played a significant role in modeling of many real areas of sciences such as physics, engineering, biology, medicine, and economics. FDDEs often cannot be solved analytically so the approximate and numerical methods should be adapted to solve these types of equations. In this paper we consider a new method of backward differentiation formula- (BDF-) type for solving FDDEs. This approach is based on the interval approximation of the true solution using the Clenshaw and Curtis formula that is based on the truncated shifted Chebyshev polynomials. It is shown that the new approach can be reformulated in an equivalent way as a Runge-Kutta method and the Butcher tableau of this method is given. Estimation of local and global truncating errors is deduced and this leads to the proof of the convergence for the proposed method. Illustrative examples of FDDEs are included to demonstrate the validity and applicability of the proposed approach.
\end{abstract}

\section{Introduction}

Fractional differential equations have been the focus of many studies due to their frequent appearance in various sciences [1-4]. Some approximate and numerical methods are used to obtain approximate solutions [5-11]. The general theory of differential equations with delays (DDEs) is widely developed and discussed in the literature [12-16]. DDEs are differential equations in which the rate of change of $y(t)$ does not depend only on the values of $y$ for the same time value but also on time values less than $t$. In the simplest case, DDEs have the form

$$
y^{\prime}(t)=f(t, y(t), y(t-\tau)), \quad t \in[0, L], \tau>0,
$$

under the following initial conditions:

$$
y(t)=\phi(t), \quad-\tau \leq t \leq 0,
$$

where $\tau$ is the delay term. DDEs arise in scientific models. These models of DDEs appeared in $[13,14,17,18]$. Recently, fractional differential equations with delay (FDDEs) gain the attention of many researchers. FDDEs have appeared also in some scientific areas [19-23]. Some approaches are built to obtain numerical and approximate solutions for FDDEs:

$$
\begin{array}{r}
D^{(\beta)} y(t)=f(t, y(t), y(t-\tau)), \quad t \in[0, L], t \geq 0, \\
m-1<\beta \leq m, \quad m=1,2,3, \ldots,
\end{array}
$$

under the following initial conditions:

$$
y(t)=\phi(t), \quad t \leq 0 .
$$

Yang and Cao [24] studied the existence and uniqueness of initial value problems for nonlinear higher fractional equations with delay by fixed point theory. The theory of fractional functional differential equation and the asymptotic properties of fractional delay differential equations are 
discussed, respectively, in [25-27]. Bhalekar and DaftardarGejji adapted the Adams Bashforth Moulton predictor corrector method (EABMPC method) [28] to solve this form of FDDEs (3)-(4), where $0<\beta \leq 1$. Besides, Wang [29] combined Adams Bashforth Moulton method with the linear interpolation method to approximate FDDEs. In addition, Wang et al. [30] introduced a numerical method based on Grunwald Letnikov definition to solve nonlinear FDDEs with constant time varying delay.

In addition to that, Morgado et al. [31] made an analysis and numerical methods for linear fractional differential equations with positive finite delay:

$$
\begin{array}{r}
D^{(\beta)} y(t)=a y(t-\tau)+b y(t)+f(t), \\
t \geq 0, \quad 0<\beta \leq 1,
\end{array}
$$

under the following initial conditions:

$$
y(t)=\phi(t), \quad t \in[-\tau, 0] .
$$

Also, Moghaddam and Mostaghim [32] discussed and introduced a novel matrix approach to fractional finite difference for solving models based on nonlinear fractional delay differential equations of the form (5)-(6). Also, Moghaddam and Mostaghim [33] developed a numerical method based on finite difference for solving fractional delay differential equations of the form (5)-(6). In [34] the authors consider a fourth-order method of BDF-type for solving stiff initialvalue problems, based on the interval approximation of the true solution by truncated Chebyshev series. It is shown that the method may be formulated as a Runge-Kutta method having stage order four.

In this paper, we focus on FDDEs with finite delay which has the following form:

$$
\begin{gathered}
D^{(\beta)} y(t)=f(t, y(t), y(t-\tau)), \quad t \in[0, L], t \geq 0, \\
m-1<\beta \leq m, \\
y(t)=\phi(t), \quad t \in[-\tau, 0] .
\end{gathered}
$$

We present fractional order Runge-Kutta method of backward differentiation formula- (BDF-) type based on approximations by truncated shifted Chebyshev series of Clenshaw and Curtis formula in the sense that we do not take an approximation of the function $f$ in the right-hand side of the differential equation, which will be integrated later, but we take a truncated Chebyshev series instead.

The structure of this paper is arranged in the following way: in Section 2, we present some necessary definitions and mathematical preliminaries of the fractional calculus theory. In Section 3, we introduce the derivation of the method and the fundamental related relations. In Section 4, we present the proposed method as a one-step recurrence formula, obtain the local truncating error, and prove that the order of the proposed method is $n \beta$ such that $n$ is the approximation order of Clenshaw and Curtis formula. In Section 5, the global truncating error of our proposed method is deduced. In Section 6, numerical examples are given to solve FDDEs and show the accuracy of the presented method. Finally, in Section 7, the paper ends with a brief conclusion and some remarks.

\section{Preliminaries and Notations}

In this section, we present some necessary definitions and mathematical preliminaries of the fractional calculus theory required for our subsequent development.

\subsection{The Caputo Fractional Derivatives}

Definition 1. The Caputo fractional derivative operator $D^{(v)}$ of order $v$ is defined in the following form:

$$
D^{(\nu)} f(x)=\frac{1}{\Gamma(m-v)} \int_{0}^{x} \frac{f^{(m)}(\xi)}{(x-\xi)^{\nu-m+1}} d \xi, \quad \nu>0,
$$

where $m-1<v \leq m, m \in \mathbb{N}, x>0$.

Similar to integer-order differentiation, the Caputo fractional derivative operator is a linear operation:

$$
D^{(v)}(\lambda p(x)+\mu q(x))=\lambda D^{(v)} p(x)+\mu D^{(v)} q(x),
$$

where $\lambda$ and $\mu$ are constants.

For Caputo's derivative we have

$$
\begin{gathered}
D^{(\nu)} C=0, \quad C \text { is a constant, } \\
D^{(v)} x^{n}= \begin{cases}0, & \text { for } n \in \mathbb{N}_{0}, n<\lceil\nu\rceil ; \\
\frac{\Gamma(n+1)}{\Gamma(n+1-\nu)} x^{n-\nu}, & \text { for } n \in \mathbb{N}_{0}, n \geq\lceil\nu\rceil .\end{cases}
\end{gathered}
$$

We use the ceiling function $\lceil\nu\rceil$ to denote the smallest integer greater than or equal to $\nu$ and $\mathbb{N}_{0}=\{0,1,2, \ldots\}$. Recall that, for $v \in \mathbb{N}$, the Caputo differential operator coincides with the usual differential operator of integer order.

For more details on fractional derivatives definitions and their properties, see [35-38].

2.2. The Definition and Properties of the Shifted Chebyshev Polynomials. The well-known Chebyshev polynomials [39] are defined on the interval $[-1,1]$ and can be determined with the aid of the following recurrence formula:

$$
\begin{array}{r}
T_{n+1}(z)=2 z T_{n}(z)-T_{n-1}(z), \\
T_{0}(z)=1, \quad T_{1}(z)=z, \quad n=1,2, \ldots .
\end{array}
$$

It is well known that $T_{n}(1)=1, T_{n}(-1)=(-1)^{n}$. The analytic form of the Chebyshev polynomials $T_{n}(z)$ of degree $n$ is given by

$$
T_{n}(z)=\sum_{i=0}^{\lfloor n / 2\rfloor}(-1)^{i} 2^{n-2 i-1} \frac{n(n-i-1) !}{(i) !(n-2 i) !} z^{n-2 i}
$$


where $\lfloor n / 2\rfloor$ denotes the integer part of $n / 2$. The orthogonality condition is

$$
\int_{-1}^{1} \frac{T_{i}(z) T_{j}(z)}{\sqrt{1-z^{2}}} d z= \begin{cases}\pi, & \text { for } i=j=0 \\ \frac{\pi}{2}, & \text { for } i=j \neq 0 \\ 0, & \text { for } i \neq j\end{cases}
$$

In order to use these polynomials on the interval $[0, L]$ we define the so-called shifted Chebyshev polynomials by introducing the change of variable $z=2 x / L-1$. The shifted Chebyshev polynomials are defined as

$$
\begin{aligned}
& T_{n}^{*}(x)=T_{n}\left(\frac{2 x}{L}-1\right), \text { where } \\
& T_{0}^{*}(x)=1, \quad T_{1}^{*}(x)=\frac{2 x}{L}-1 .
\end{aligned}
$$

The analytic form of the shifted Chebyshev polynomial $T_{n}^{*}(x)$ of degree $n$ is given by

$$
T_{n}^{*}(x)=n \sum_{k=0}^{n}(-1)^{n-k} \frac{(n+k-1) ! 2^{2 k}}{(n-k) !(2 k) ! L^{k}} x^{k},
$$

where $T_{n}^{*}(0)=(-1)^{n}$ and $T_{n}^{*}(L)=1$. The orthogonality condition of these polynomials is

$$
\int_{0}^{L} T_{j}^{*}(x) T_{k}^{*}(x) w(x) d x=\delta_{j k} h_{k}
$$

where the weight function $w(x)=1 / \sqrt{L x-x^{2}}, h_{k}=\left(b_{k} / 2\right) \pi$, with $b_{0}=2, b_{k}=1, k \geq 1$.

The function $y(x)$, which belongs to the space of square integrable in $[0, L]$, may be expressed in terms of shifted Chebyshev polynomials as

$$
y(x)=\sum_{n=0}^{\infty} c_{n} T_{n}^{*}(x)
$$

where the coefficients $c_{n}$ are given by

$$
c_{n}=\frac{1}{h_{n}} \int_{0}^{L} y(x) T_{n}^{*}(x) w(x) d x, \quad n=0,1,2, \ldots
$$

The well-known shifted Chebyshev polynomials of the first kind of degree $n$ are defined on the interval $[0, L]$ as in $(15)$. We choose the grid (interpolation) points to be the ChebyshevGauss Lobatto points associated with the interval $[0, L], x_{r}=$ $L / 2-(L / 2) \cos (\pi r / N), r=0,1, \ldots, N$. These grids can be written as $L=x_{N}<x_{N-1}<\cdots<x_{1}<x_{0}=0$.

Clenshaw and Curtis [40] introduced an approximation of the function $y(x)$, and we reformulate it to be used on the shifted Chebyshev polynomials as follows:

$$
y_{N}(x)=\sum_{n=0}^{N}{ }^{\prime \prime} a_{n} T_{n}^{*}(x), \quad a_{n}=\frac{2}{N} \sum_{r=0}^{N} y\left(x_{r}\right) T_{n}^{*}\left(x_{r}\right) \text {. }
$$

The summation symbol with double primes denotes a sum with both first and last terms halved.

\section{Derivation of the Numerical Approximation Scheme}

Let us consider the initial value problem for arbitrary order fractional differential equation with delay of the form

$$
\begin{aligned}
& D^{(\beta)} y(t)=f(t, y(t), y(t-\tau)), \\
& t \in[0, L], \quad t \geq 0, \quad y(t)=\phi(t), t \in[-\tau, 0],
\end{aligned}
$$

such that $m-1<\beta \leq m$ and $m$ is an integer.

Yang and Cao studied the existence and uniqueness of these types of (20) using the fixed point theory [24]. Now, let $y_{s}$ be an approximation of the theoretical solution $y(t)$ at $t_{s}$. We are interested in obtaining a numerical approximation at the point $t_{s+1}=t_{s}+h$ such that $h$ is the step size.

If we rewrite the solution $y(t)$ in the interval $\left[t_{s}, t_{s+1}\right]$ in terms of a new variable $\alpha$ defined by

$$
t=t_{s}+\frac{h \alpha}{L}, \quad \alpha \in[0, L]
$$

we can approximate the solution of FDDEs expressed in the form

$$
y(t)=y\left(t_{s}+\frac{h \alpha}{L}\right)=\bar{y}(\alpha)
$$

Approximate (22) in terms of Clenshaw and Curtis finite sum approximations as follows:

$$
\bar{y}(\alpha)=\sum_{n=0}^{N}{ }^{\prime \prime} a_{n} T_{n}^{*}(\alpha), \quad a_{n}=\frac{2}{N} \sum_{r=0}^{N} y\left(t_{s}+\zeta_{r} h\right) T_{n}^{*}\left(\alpha_{r}\right),
$$

such that

$$
\begin{gathered}
\zeta_{r}=\frac{\alpha_{r}}{L}, \quad \alpha_{r}=\frac{L}{2}-\frac{L}{2} \cos \left(\frac{\pi r}{N}\right), \\
D_{t}^{(\beta)} \bar{y}(\alpha) \\
=\frac{2}{N} \sum_{n=0}^{N} \sum_{r=0}^{N} \sum^{\prime \prime} y\left(t_{s}+\zeta_{r} h\right) T_{n}^{*}\left(\alpha_{r}\right) D_{t}^{(\beta)} T_{n}^{*}(\alpha(t)) .
\end{gathered}
$$


Using the analytic form of shifted Chebyshev polynomials (15) and the properties of Caputo fractional derivatives (10) gives

$$
\begin{aligned}
& D_{t}^{(\beta)} T_{n}^{*}(\alpha(t)) \\
& =\sum_{k=0}^{n} \frac{n(-1)^{(n-k)} 2^{(2 k)}(n+k-1) !}{(n-k) !(2 k) ! L^{k}} D_{t}^{(\beta)}\left(\left(\frac{L}{h} t-\frac{L}{h} t_{s}\right)^{k}\right) \\
& =\sum_{k=\lceil\beta\rceil}^{n} \frac{n(-1)^{(n-k)} 2^{(2 k)}(n+k-1) ! \Gamma(k+1)}{(n-k) !(2 k) ! L^{k} \Gamma(k+1-\beta)} \\
& \quad \cdot\left(\left(\frac{L}{h}\right)^{(\beta)}\left(\frac{L}{h}\left(t-t_{s}\right)\right)^{k-\beta}\right) \\
& =\sum_{k=\lceil\beta\rceil}^{n} \frac{n(-1)^{(n-k)} 2^{(2 k)}(n+k-1) ! \Gamma(k+1) L^{\beta}}{(n-k) !(2 k) ! L^{k} \Gamma(k+1-\beta) h^{\beta}} \alpha^{k-\beta} .
\end{aligned}
$$

Now, $\alpha^{k-\beta}$ can be expressed approximately in terms of shifted Chebyshev series as follows:

$$
\alpha^{k-\beta} \cong \sum_{j=0}^{N} c_{k j} T_{j}^{*}(\alpha),
$$

where $c_{k j}$ is obtained from (18) as clarified by Doha et al. [5],

$$
c_{k j}=\left\{\begin{array}{l}
\frac{1}{\sqrt{\pi}} \frac{L^{k-\beta} \Gamma(k-\beta+1 / 2)}{\Gamma(k-\beta+1)}, \quad \text { for } j=0 ; \\
\frac{j L^{k-\beta}}{\sqrt{\pi}} \\
\cdot \sum_{r=0}^{j} \frac{(-1)^{j-r}(j+r-1) ! 2^{2 r+1} \Gamma(k+r-\beta+1 / 2)}{(j-r) !(2 r) ! \Gamma(k+r-\beta+1)} \\
\text { for } j=1,2, \ldots
\end{array}\right.
$$

From (27)-(29), we get

$$
\begin{array}{r}
D_{t}^{(\beta)} T_{n}^{*}(\alpha(t))=\frac{1}{h^{\beta}} \sum_{j=0}^{\infty} \omega_{\beta}(n, j) T_{j}^{*}(\alpha), \\
n=\lceil\beta\rceil,\lceil\beta\rceil+1, \ldots,
\end{array}
$$

where $\omega_{\beta}(n, j)=\sum_{k=\lceil\beta\rceil}^{n} \psi_{n, j, k}$,

$$
\psi_{n, j, k}=\left\{\begin{array}{c}
\frac{n(-1)^{n-k}(n+k-1) ! 2^{2 k} k ! \Gamma(k-\beta+1 / 2)}{L^{\beta}(n-k) !(2 k) ! \sqrt{\pi} \Gamma(k-\beta+1)^{2}}, \\
\text { for } j=0 ; \\
\frac{(-1)^{n-k} n j(n+k-1) ! 2^{2 k+1} k !}{L^{\beta}(n-k) !(2 k) ! \Gamma(k-\beta+1) \sqrt{\pi}} \\
\cdot \sum_{r=0}^{j} \frac{(-1)^{j-r}(j+k-1) ! 2^{2 r} \Gamma(k+r-\beta+1 / 2)}{(j-r) !(2 r) ! \Gamma(k+r-\beta+1)} \\
\text { for } j=1,2, \ldots
\end{array}\right.
$$

Then, $\psi_{n, j, k}$ can be put in the following form:

$$
\begin{aligned}
\psi_{n, j, k} & \\
= & (-1)^{n-k} 2 n(n+k-1) ! \Gamma\left(k-\beta+\frac{1}{2}\right) \\
& \cdot\left(b_{j} L^{\beta} \Gamma\left(k+\frac{1}{2}\right)(n-k) ! \Gamma(k-\beta-j+1)\right. \\
& \cdot \Gamma(k+j-\beta+1))^{-1}, \quad j=0,1, \ldots
\end{aligned}
$$

Then,

$$
\begin{gathered}
D_{t}^{(\beta)} T_{n}^{*}(\alpha(t)) \\
=\frac{1}{h^{\beta}} \sum_{j=0}^{N} \sum_{k=\lceil\beta\rceil}^{n}\left((-1)^{(n-k)} 2 n(n+k-1) ! \Gamma\left(k-\beta+\frac{1}{2}\right)\right. \\
\cdot\left(b_{j} \Gamma\left(k+\frac{1}{2}\right)(n-k) ! \Gamma(k-\beta+j+1)\right. \\
\left.\cdot \Gamma(k-\beta-j+1))^{-1}\right) T_{j}^{*}(\alpha) .
\end{gathered}
$$

From (23) and (33), we obtain the following approximate equation:

$$
\begin{aligned}
& f(t, y(t), y(t-\tau)) \\
& \quad=\frac{4}{N h^{\beta}} \sum_{n=\lceil\beta\rceil}^{N} \sum_{r=0}^{N} \sum_{j=0}^{N} \sum_{k=\lceil\beta\rceil}^{n} \frac{(-1)^{(n-k)} n(n+k-1) ! \Gamma(k-\beta+1 / 2) y\left(t_{s}+\zeta_{r} h\right) T_{n}^{*}\left(\alpha_{r}\right)}{b_{j} \Gamma(k+1 / 2)(n-k) ! \Gamma(k-\beta+j+1) \Gamma(k-\beta-j+1)} T_{j}^{*}(\alpha) .
\end{aligned}
$$


Evaluating the formula (34) at $\alpha_{\ell}=L / 2-(L / 2) \cos (\pi \ell / N)$, $\ell=1,2, \ldots, N$ gives an implicit system of algebraic equations

$$
\begin{aligned}
f & \left(t_{s}+\zeta_{\ell} h, y\left(t_{s}+\zeta_{\ell} h\right), y\left(t_{s}+\zeta_{\ell} h-\tau\right)\right) \\
& =\frac{4}{N h^{\beta}} \sum_{n=\lceil\beta\rceil}^{N}{ }^{\prime \prime} \sum_{r=0}^{N} \prime \sum_{j=0}^{N} \sum_{k=\lceil\beta\rceil}^{n} \frac{(-1)^{(n-k)} n(n+k-1) ! \Gamma(k-\beta+1 / 2) y\left(t_{s}+\zeta_{r} h\right) T_{n}^{*}\left(\alpha_{r}\right)}{b_{j} \Gamma(k+1 / 2)(n-k) ! \Gamma(k-\beta+j+1) \Gamma(k-\beta-j+1)} T_{j}^{*}\left(\alpha_{\ell}\right) .
\end{aligned}
$$

It can be observed that, for any delayed term $\tau,\left(t_{s}+\zeta_{\ell} h-\right.$ $\tau$ ) may not be a grid point $t_{\ell}$ for any $\ell$. So, we establish the approximation for the delayed function $y(t-\tau)$ as follows:

$$
d_{\ell}=y\left(t_{\ell}-\tau\left(t_{\ell}\right)\right)=y\left(t_{s}+\zeta_{\ell} h-\tau\left(t_{s}+\zeta_{\ell} h\right)\right) .
$$

So, we define the delayed term as $\tau=\left(m_{s}+\delta_{\ell}\right) h$ such that $0 \leq \delta_{\ell}<1$ and $m_{s}$ is a positive integer. As $t_{s+1}=t_{s}+h$, then

$$
d_{\ell}=y\left(t_{0}+\left(s-m_{s}\right) h+\left(\zeta_{\ell}-\delta_{\ell}\right) h\right) .
$$

Then, we have two different cases.

The First Case $\left(\delta_{\ell}=0\right)$. Consider

$$
d_{\ell}= \begin{cases}y\left(t_{s-m_{s}}+\zeta_{\ell} h\right), & \text { for } s>m_{s} \\ \Lambda_{\ell}, & \text { for } s \leq m_{s}\end{cases}
$$

such that

$$
\Lambda_{\ell}= \begin{cases}y\left(t_{0}+\zeta_{\ell} h\right), & \text { for } s=m_{s} \\ y\left(t_{0}\right), & \text { for } s+\zeta_{\ell}=m_{s} \\ \phi\left(t_{\ell}-\tau\left(t_{\ell}\right)\right), & \text { for } s+\zeta_{\ell}<m_{s}\end{cases}
$$

The Second Case $\left(0<\delta_{\ell}<1\right)$. In this case, $y(t-\tau)$ is not a grid point. To overcome this, we introduce the following approximation:

$$
\begin{aligned}
& d_{\ell}=y\left(t_{0}+\left(s-m_{s}-1\right) h+\zeta_{\ell} h-\left(1-\delta_{\ell}\right) h\right) \begin{array}{l}
\text { From (35)-(42), we have the following nume } \\
\text { tion scheme: }
\end{array} \\
&\left.h^{\beta} f\left(t_{s}+\zeta_{\ell} h, y\left(t_{s-m_{s}-1}+\zeta_{\ell} h\right)+\left(1-\delta_{\ell}\right) h\right), d_{\ell}\right) \\
&=\frac{4}{N} \sum_{n=\lceil\beta\rceil}^{N}{ }^{\prime \prime} \sum_{r=0}^{N} \sum_{j=0}^{N} \sum_{k=\lceil\beta\rceil}^{n} \frac{(-1)^{(n-k)} n(n+k-1) ! \Gamma(k-\beta+1 / 2) y\left(t_{s}+\zeta_{r} h\right) T_{n}^{*}\left(\alpha_{r}\right)}{b_{j} \Gamma(k+1 / 2)(n-k) ! \Gamma(k-\beta+j+1) \Gamma(k-\beta-j+1)} T_{j}^{*}\left(\alpha_{\ell}\right),
\end{aligned}
$$

$$
\begin{aligned}
\simeq & y\left(t_{s-m_{s}-1}+\zeta_{\ell} h\right) \\
& +\left(1-\delta_{\ell}\right) h y^{\prime}\left(t_{s-m_{s}-1}+\zeta_{\ell} h\right)+O\left(h^{2}\right) \\
\simeq & y\left(t_{s-m_{s}-1}+\zeta_{\ell} h\right)+\left(1-\delta_{\ell}\right) h \\
& \cdot\left[\frac{y\left(t_{s-m_{s}-1}+\zeta_{\ell} h+h\right)-y\left(t_{s-m_{s}-1}+\zeta_{\ell} h\right)}{h}\right] \\
\simeq & \left(1-\delta_{\ell}\right) y\left(t_{s-m_{s}}+\zeta_{\ell} h\right)+\delta_{\ell} y\left(t_{s-m_{s}-1}+\zeta_{\ell} h\right) .
\end{aligned}
$$

Then, we have

$$
d_{\ell}= \begin{cases}\left(1-\delta_{\ell}\right) y\left(t_{s-m_{s}}+\zeta_{\ell} h\right) & \\ +\delta_{\ell} y\left(t_{s-m_{s}-1}+\zeta_{\ell} h\right), & \text { for } s>m_{s} ; \\ \left(1-\delta_{\ell}\right) y\left(t_{0}+\zeta_{\ell} h\right)+\delta_{\ell} \phi\left(t_{\ell}-\tau\left(t_{\ell}\right)\right), & \text { for } s=m_{s} \\ \hbar_{\ell}, & \text { for } s<m_{s},\end{cases}
$$

such that

$$
\hbar_{\ell}= \begin{cases}\left(1-\delta_{\ell}\right) y\left(t_{0}\right)+\delta_{\ell} \phi\left(t_{\ell}-\tau\left(t_{\ell}\right)\right), & \text { for } s+\zeta_{\ell}=m_{s} \\ \phi\left(t_{\ell}-\tau\left(t_{\ell}\right)\right), & \text { for } s+\zeta_{\ell}<m_{s}\end{cases}
$$

From (35)-(42), we have the following numerical approxima- where the unknowns are the values of the solution at the intermediate points $y\left(t_{s}+\zeta_{\ell} h\right)$.

For DDEs $(\beta=1)$, explicitly, if we put $N=4$ in (43), the algebraic system will have the following form:

$$
\begin{aligned}
h \mathbf{f}_{1}= & -(2+\sqrt{2}) y\left(t_{s}\right)+\sqrt{2} y\left(t_{s}+\zeta_{1} h\right) \\
& +2 \sqrt{2} y\left(t_{s}+\zeta_{2} h\right)-\sqrt{2} y\left(t_{s}+\zeta_{3} h\right) \\
& +(2-\sqrt{2}) y\left(t_{s}+\zeta_{4} h\right),
\end{aligned}
$$

$$
\begin{aligned}
h \mathbf{f}_{2}= & 1 y\left(t_{s}\right)-2 \sqrt{2} y\left(t_{s}+\zeta_{1} h\right)+0 y\left(t_{s}+\zeta_{2} h\right) \\
& +2 \sqrt{2} y\left(t_{s}+\zeta_{3} h\right)-1 y\left(t_{s}+\zeta_{4} h\right) \\
h \mathbf{f}_{3}= & -(2-\sqrt{2}) y\left(t_{s}\right)+\sqrt{2} y\left(t_{s}+\zeta_{1} h\right) \\
& -2 \sqrt{2} y\left(t_{s}+\zeta_{2} h\right)-\sqrt{2} y\left(t_{s}+\zeta_{3} h\right) \\
& +(2+\sqrt{2}) y\left(t_{s}+\zeta_{4} h\right),
\end{aligned}
$$




$$
\begin{aligned}
h \mathbf{f}_{4}= & 1 y\left(t_{s}\right)-(8-4 \sqrt{2}) y\left(t_{s}+\zeta_{1} h\right)+4 y\left(t_{s}+\zeta_{2} h\right) \\
& -(8+4 \sqrt{2}) y\left(t_{s}+\zeta_{3} h\right)+11 y\left(t_{s}+\zeta_{4} h\right) .
\end{aligned}
$$

For FDDEs $(\beta=1 / 2)$, also, if we put $N=4$ in (43), the algebraic system will have the following form:

$$
\begin{aligned}
h^{0.5} \mathbf{f}_{1}= & -(\sqrt{2}(335+182 \sqrt{2}) a) y\left(t_{s}\right) \\
& +((280+320 \sqrt{2}) a) y\left(t_{s}+\zeta_{1} h\right) \\
& -(12 \sqrt{2}(1-7 \sqrt{2}) a) y\left(t_{s}+\zeta_{2} h\right) \\
& -((56+16 \sqrt{2}) a) y\left(t_{s}+\zeta_{3} h\right) \\
& +((-28+43 \sqrt{2}) a) y\left(t_{s}+\zeta_{4} h\right), \\
h^{0.5} \mathbf{f}_{2}= & -\left(\frac{71 \sqrt{2}}{d}\right) y\left(t_{s}\right) \\
& -\left(\frac{(168+40 \sqrt{2})}{d}\right) y\left(t_{s}+\zeta_{1} h\right) \\
& +\left(\frac{180 \sqrt{2}}{d}\right) y\left(t_{s}+\zeta_{2} h\right) \\
& +((168+152 \sqrt{2}) c) y\left(t_{s}+\zeta_{3} h\right) \\
& +\left(\frac{(168-40 \sqrt{2})}{d}\right) y\left(t_{s}+\zeta_{3} h\right) \\
& +\left(\left(t_{s} h\right),\right. \\
& -(168-152 \sqrt{2}) c) y\left(t_{s}+\zeta_{1} h\right) \\
& -\left(\frac{(29 \sqrt{2})}{d}\right) 8 y\left(t_{s}+\zeta_{4} h\right), \\
h^{0.5} \mathbf{f}_{3}= & -(\sqrt{2}(335-182 \sqrt{2}) b) y\left(t_{s}\right) \\
& +((56-16 \sqrt{2}) b) y\left(t_{s}+\zeta_{1} h\right) b y\left(t_{s}+\zeta_{2} h\right) \\
& \left.+\zeta_{2} h\right) \\
& +320 \sqrt{2}) b y\left(t_{s}+\zeta_{3} h\right) \\
& +(4 \sqrt{2} c) b) y\left(t_{s}+\zeta_{4} h\right), \\
& \left.+t_{s}\right) \\
& +(120) \\
&
\end{aligned}
$$

such that

$$
\begin{array}{rlrl}
a=\frac{1}{105} \sqrt{\frac{2}{\pi}} \sin \left(\frac{\pi}{8}\right), & b & =\frac{1}{105} \sqrt{\frac{2}{\pi}} \cos \left(\frac{\pi}{8}\right), \\
c & =\frac{1}{105} \sqrt{\frac{2}{\pi}}, \quad d=105 \sqrt{\pi} .
\end{array}
$$

For the sake of simplicity, we take this abbreviation

$$
\mathbf{f}_{\ell}=f\left(t_{s}+\zeta_{\ell} h, y\left(t_{s}+\zeta_{\ell} h\right), d_{\ell}\right), \quad \ell=1,2,3,4 .
$$

Solving this system, we obtain in particular the required value at the final point on the interval $y\left(t_{s}+\zeta_{4} h\right)=y\left(t_{s}+h\right)=$ $y_{s+1}$. If we repeat this procedure along the integration interval $[0, L]$, a discrete solution for the problem in (20) will be deduced.

\section{One-Step Recurrence Formula Expression and the Local Truncating Error}

The method in (43) at $N=4$ can be expressed by one step recurrence formula:

$$
C Y_{s}=B Y_{s-1}+h^{\beta} F_{s},
$$

where

$$
\begin{gathered}
F_{s}=\left[\mathbf{f}_{1}, \mathbf{f}_{2}, \mathbf{f}_{3}, \mathbf{f}_{4}\right]^{T}, \\
Y_{s}=\left[y\left(t_{s}+\zeta_{1} h\right), y\left(t_{s}+\zeta_{2} h\right), y\left(t_{s}+\zeta_{3} h\right), y\left(t_{s}+\zeta_{4} h\right)\right]^{T}, \\
Y_{s-1}=\left[y\left(t_{s-1}+\zeta_{1} h\right), y\left(t_{s-1}+\zeta_{2} h\right), y\left(t_{s-1}+\zeta_{3} h\right),\right. \\
\left.y\left(t_{s-1}+\zeta_{4} h\right)\right]^{T} .
\end{gathered}
$$

Remark 2. Consider

$$
\begin{gathered}
\quad y\left(t_{s}-\zeta_{4-\ell} h\right)=y\left(t_{s-1}+\zeta_{\ell} h\right), \quad \ell=1,2,3,4, \\
Y_{s-1} \\
=\left[y\left(t_{s}+\zeta_{3} h\right), y\left(t_{s}+\zeta_{2} h\right), y\left(t_{s}+\zeta_{1} h\right), y\left(t_{s}+\zeta_{0} h\right)\right]^{T} .
\end{gathered}
$$

For DDEs $(\beta=1)$, the constant matrices $B$ and $C$ are given by

$$
\begin{gathered}
B=\left(\begin{array}{cccc}
0 & 0 & 0 & 2+\sqrt{2} \\
0 & 0 & 0 & 1 \\
0 & 0 & 0 & 2-\sqrt{2} \\
0 & 0 & 0 & -1
\end{array}\right), \\
C=\left(\begin{array}{cccc}
\sqrt{2} & 2 \sqrt{2} & -\sqrt{2} & 2-\sqrt{2} \\
-2 \sqrt{2} & 0 & 2 \sqrt{2} & -1 \\
\sqrt{2} & -2 \sqrt{2} & -\sqrt{2} & 2+\sqrt{2} \\
-8+4 \sqrt{2} & 4 & -8-4 \sqrt{2} & 11
\end{array}\right),
\end{gathered}
$$


which have a completely agreement with the work done by Ramos and Vigo-Aguiar [34].
For FDDEs $(\beta=0.5)$, the constant matrices $B$ and $C$ are also given by

$$
\begin{gathered}
B=\left(\begin{array}{cccc}
0 & 0 & 0 & \sqrt{2}(335+182 \sqrt{2}) a \\
0 & 0 & 0 & \frac{71 \sqrt{2}}{d} \\
0 & 0 & 0 & \sqrt{2}(335-182 \sqrt{2}) b \\
0 & 0 & 0 & 43 \sqrt{2} c
\end{array}\right), \\
C=\left(\begin{array}{cccc}
(280+320 \sqrt{2}) a & -12 \sqrt{2}(1-7 \sqrt{2}) a & -(56+16 \sqrt{2}) a & (-28+43 \sqrt{2}) a \\
\frac{(-168-40 \sqrt{2})}{d} & \frac{180 \sqrt{2}}{d} & \frac{(168-40 \sqrt{2})}{d} & \frac{(-29 \sqrt{2})}{d} \\
(56-16 \sqrt{2}) b & -12 \sqrt{2}(1+7 \sqrt{2}) b & (-280+320 \sqrt{2}) b & (28+43 \sqrt{2}) b \\
(168-152 \sqrt{2}) c & 12 \sqrt{2} c & (-168-152 \sqrt{2}) c & 335 \sqrt{2} c
\end{array}\right) .
\end{gathered}
$$

As the matrix $C$ is nonsingular, multiplying the formula

by the inverse matrix $C^{-1}$ yields

$$
Y_{s}=C^{-1} B Y_{s-1}+h^{\beta} C^{-1} F_{s} .
$$

Remark 3. The product $C^{-1} B$ is a square matrix of dimension 4 on the following form:

$$
\left(\begin{array}{llll}
0 & 0 & 0 & 1 \\
0 & 0 & 0 & 1 \\
0 & 0 & 0 & 1 \\
0 & 0 & 0 & 1
\end{array}\right) .
$$

Remark 4. The method expressed finally by (53) can be considered as a Runge-Kutta method where the Butcher tableau [41] is given by the following.

(i) For DDEs $(\beta=1)$, see Table 1 .

When we compare this tableau with the tableau obtained by Ramos and Vigo-Aguiar [34], we find a complete agreement.

(ii) For FDDEs $(\beta=0.5)$, see Table 2 .

The contents of Butcher tableau at $\beta=0.5$ can be written also in terms of fractions but it will be complicated. So, we write it in decimal form.

In the names of Remark 4, we can say that the theory of Runge-Kutta methods may be applied here. For our approach, when we deal with DDEs $(\beta=1)$, the Butcher conditions for order four are verified. So, this method has the fourth order for DDEs.
Consider the difference operators associated with each stage in our method as follows:

$$
\begin{aligned}
& L_{i}(y(t), h) \\
& =y\left(t_{s}+\zeta_{i} h\right)-y\left(t_{s}\right)-h^{\beta} \sum_{j=1}^{N} c_{i j} y^{(\beta)}\left(t_{s}+\zeta_{i} h\right), \\
& \quad i=1,2,3,4, \\
& L_{i}(y(t), h) \\
& =y\left(t_{s}+\zeta_{i} h\right)-y\left(t_{s}\right) \\
& \quad-h^{\beta} \sum_{j=1}^{N} c_{i j} f\left(t_{s}+\zeta_{i} h, y\left(t_{s}+\zeta_{i} h\right), d_{i}\right),
\end{aligned}
$$$$
i=1,2,3,4 \text {, }
$$

and using the fractional Taylor series about $t_{s}$, we obtain

$$
\begin{gathered}
y\left(t_{s}+\zeta_{i} h\right) \\
=y\left(t_{s}\right)+y^{(\beta)}\left(t_{s}\right) \frac{\left(\zeta_{i} h\right)^{\beta}}{\Gamma(\beta+1)}+y^{(2 \beta)}\left(t_{s}\right) \frac{\left(\zeta_{i} h\right)^{2 \beta}}{\Gamma(2 \beta+1)} \\
+\cdots+y^{((N+1) \beta)}\left(t_{s}+\theta \zeta_{i} h\right) \frac{\left(\zeta_{i} h\right)^{(N+1) \beta}}{\Gamma((N+1) \beta+1)}, \\
f\left(t_{s}+\zeta_{i} h, y\left(t_{s}+\zeta_{i} h\right), d_{i}\right) \\
=\mathbf{f}_{i}\left(\zeta_{i} h\right)=\mathbf{f}_{i}(0)+\mathbf{f}_{i}^{(\beta)}(0) \frac{\left(\zeta_{i} h\right)^{\beta}}{\Gamma(\beta+1)} \\
+\mathbf{f}_{i}^{(2 \beta)}(0) \frac{\left(\zeta_{i} h\right)^{2 \beta}}{\Gamma(2 \beta+1)} \\
+\cdots+\mathbf{f}_{i}^{(N \beta)}\left(\theta \zeta_{i} h\right) \frac{\left(\zeta_{i} h\right)^{N \beta}}{\Gamma(N \beta+1)} .
\end{gathered}
$$


TABLE 1

\begin{tabular}{lcccc}
\hline$\alpha_{1}$ & $(1 / 384)(88-4 \sqrt{2})$ & $(1 / 384)(40-64 \sqrt{2})$ & $(1 / 384)(88-28 \sqrt{2})$ & $-1 / 16$ \\
$\alpha_{2}$ & $(1 / 384)(64+48 \sqrt{2})$ & $1 / 6$ & $(1 / 384)(64-48 \sqrt{2})$ & 0 \\
$\alpha_{3}$ & $(1 / 384)(88+28 \sqrt{2})$ & $(1 / 384)(40+64 \sqrt{2})$ & $(1 / 384)(88+4 \sqrt{2})$ & $-1 / 16$ \\
$\alpha_{4}$ & $1 / 3$ & $1 / 3$ & $1 / 3$ & 0 \\
\hline
\end{tabular}

TABLE 2

\begin{tabular}{lllll}
\hline$\alpha_{1}$ & 0.414917 & -0.0779 & 0.0557512 & -0.0254207 \\
$\alpha_{2}$ & 0.317636 & 0.528742 & -0.101593 & 0.0415419 \\
$\alpha_{3}$ & 0.172975 & 0.420603 & 0.495111 & -0.0645867 \\
$\alpha_{4}$ & 0.169572 & 0.313329 & 0.409383 & 0.221557 \\
\hline & 0.169572 & 0.313329 & 0.409383 & 0.221557 \\
\hline
\end{tabular}

Theorem 5. If the proposed Runge-Kutta method with shifted Chebyshev polynomials (48) is of order $N \beta$ and if all partial fractional derivatives of $f(t, y(t), y(t-\tau))$ up to order $N \beta$ exist and are continuous, then the local error of (56) admits the rigorous bound:

$$
\begin{aligned}
& \left\|L_{i}\right\| \\
& \leq h^{(N+1) \beta}\left(\frac{1}{\Gamma((N+1) \beta)} \max _{\epsilon \in[0,1]}\left\|y^{((N+1) \beta)}\left(t_{s}+\epsilon \zeta_{i} h\right)\right\|\right. \\
& \\
& \left.+\frac{1}{\Gamma((N+1) \beta)} \sum_{j=1}^{N}\left|c_{i j}\right| \max _{\epsilon \in[0,1]}\left\|\mathbf{f}_{i}^{((N) \beta)}\left(\epsilon \zeta_{i} h\right)\right\|\right),
\end{aligned}
$$

and so

$$
\left\|L_{i}\right\| \leq C h^{(N+1) \beta} .
$$

Proof. Because of the order conditions and using (57), the first terms in the $h$ expansion of (56) vanish. Then (58) is obtained and the proof is complete.

Under the assumption of Theorem 5, we see that the expressions (58) and (59) are bounded by a constant independent of $h^{(N+1) \beta}$.

Remark 6. The proposed Runge-Kutta method with shifted Chebyshev polynomials (48) has order $N \beta$ for sufficiently smooth problem FDDEs (20) if (59) is achieved.

\section{Estimation of the Global Truncating Error}

The global error is the error of the approximated solution after many steps.

Theorem 7. Suppose that $y(t)$ is the exact solution of the system of FDDEs (20) and that $\nu(t)$ is the approximate solution. If the following inequalities are held:

$$
\begin{gathered}
\left\|\nu\left(t_{0}\right)-y\left(t_{0}\right)\right\| \leq \phi\left(t_{0}\right), \quad t_{0} \in[-\tau, 0], \\
\left\|D_{t}^{(\beta)} \nu(t)-f(t, \nu(t), \nu(t-\tau))\right\| \leq \varepsilon,
\end{gathered}
$$

$$
\begin{aligned}
& \|f(t, v(t), v(t-\tau))-f(t, y(t), y(t-\tau))\| \\
& \quad \leq l\|v(t)-y(t)\|
\end{aligned}
$$

then for $t \geq t_{0}$, one has the following error estimate:

$$
\begin{aligned}
\| \nu(t) & -y(t) \| \\
\leq & \phi(t) \exp \left(l\left(\frac{t^{\alpha}}{\Gamma(\alpha+1)}-\frac{t_{0}^{\alpha}}{\Gamma(\alpha+1)}\right)\right) \\
& +\frac{\varepsilon}{l}\left(\exp \left(l\left(\frac{t^{\alpha}}{\Gamma(\alpha+1)}-\frac{t_{0}^{\alpha}}{\Gamma(\alpha+1)}\right)\right)-1\right) .
\end{aligned}
$$

Proof. For any chosen norm, we investigate the error estimated by

$$
m(t)=\|v(t)-y(t)\|,
$$

and now, we try to estimate its natural growth.

Operate on both sides of (64) by the Caputo fractional derivative operator $D_{t}^{(\beta)}$ and using the triangle inequality yields

$$
\begin{aligned}
D_{t}^{(\beta)} m(t) \leq & \left\|D_{t}^{(\beta)} v(t)-f(t, v(t), v(t-\tau))\right\| \\
& +\|f(t, v(t), v(t-\tau))-f(t, y(t), y(t-\tau))\|,
\end{aligned}
$$

from Lipchitz condition (62), and we introduce $\delta(t)=$ $\left\|D_{t}^{(\beta)} v(t)-f(t, v(t), v(t-\tau))\right\|$ which is called the defect of the approximate solution $\nu(t)$ such that $\delta(t) \leq \varepsilon$; we have

$$
\begin{aligned}
D_{t}^{(\beta)} m(t) & \leq \delta(t)+\operatorname{lm}(t), \quad \delta(t) \leq \varepsilon, \\
m(t) & =\phi(t), \quad \forall t \in[-\tau, 0] .
\end{aligned}
$$

Now, we are about to solve instead of (67) the fractional differential equation and conclude that

$$
\begin{gathered}
m(t) \leq u(t), \\
D_{t}^{(\beta)} u(t)=l u(t)+\varepsilon, \\
u(t)=\phi(t), \quad \forall t \in[-\tau, 0] .
\end{gathered}
$$

To solve (69)-(70), we use the fractional complex transform $T=t^{\beta} / \Gamma(\beta+1)$ and the modified Riemann-Liouville fractional derivative defined by

$$
D_{t}^{(\beta)} u(t)=\frac{1}{\Gamma(n-\beta)} \frac{d^{n}}{d t^{n}} \int_{0}^{t}(t-\tau)^{n-\beta}(u(\tau)-u(0)) d \tau,
$$

where $n-1 \leq \beta<n$ and $n \geq 1$. 
Modified Riemann-Liouville fractional derivative has the following property:

$$
D_{t}^{(\beta)} u(T(t))=u_{T}^{\prime}(T) T^{(\beta)}(t) .
$$

Then (69)-(70) are transformed to

$$
\frac{d u}{d T}=l u(T)+\varepsilon, \quad u(T)=\phi(T), \quad \forall T \in[-\tau, 0] .
$$

The exact solution for (73) can be written as

$$
u(T)=\phi(t) \exp \left(l\left(T-T_{0}\right)\right)+\frac{\varepsilon}{l}\left(\exp \left(l\left(T-T_{0}\right)\right)-1\right) .
$$

Then the solution can be written using the original variables as follows:

$$
\begin{aligned}
u(t)= & \phi(t) \exp \left(l\left(\frac{t^{\alpha}}{\Gamma(\alpha+1)}-\frac{t_{0}^{\alpha}}{\Gamma(\alpha+1)}\right)\right) \\
& +\frac{\varepsilon}{l}\left(\exp \left(l\left(\frac{t^{\alpha}}{\Gamma(\alpha+1)}-\frac{t_{0}^{\alpha}}{\Gamma(\alpha+1)}\right)\right)-1\right) .
\end{aligned}
$$

A combination of (68) and (75) yields the desired result (63)

Theorem 8. Let $U$ be a neighborhood of $\left(t, y(t) \mid t_{0} \leq t \leq t_{N}\right)$ where $y(t)$ is the exact solution of (20). Suppose that in $U$

$$
\left\|\frac{d f}{d y}\right\| \leq l, \quad \mu\left(\frac{d f}{d y}\right) \leq l
$$

and that the local error estimates (59) are valid in $U$. Then the global error $E$ can be estimated by

$\|E\|$

$$
\begin{aligned}
\leq & \frac{\Gamma(\beta) h^{N \beta}}{t_{N}^{\beta-1}} \\
& \cdot \frac{C^{\prime}}{l}\left(\left(\frac{t_{N}}{t_{0}}\right)^{1-\beta} \exp \left(l\left(\frac{t_{N}^{\beta}}{\Gamma(\beta+1)}-\frac{t_{0}^{\beta}}{\Gamma(\beta+1)}\right)-1\right)\right),
\end{aligned}
$$

where $h=\max _{1<s \leq N} h_{s-1}$,

$$
C^{\prime}= \begin{cases}C, & \text { for } l \geq 0 \\ C \exp \left(-l h^{\beta}\right), & \text { for } l<0 .\end{cases}
$$

And $h$ is small enough for the numerical solution to remain in $U$.

Proof. Our task is to estimate the global error

$$
E=y\left(t_{N}\right)-y_{N}
$$

and this estimate is found along $N-s$ steps of the numerical method (see Figure 1).

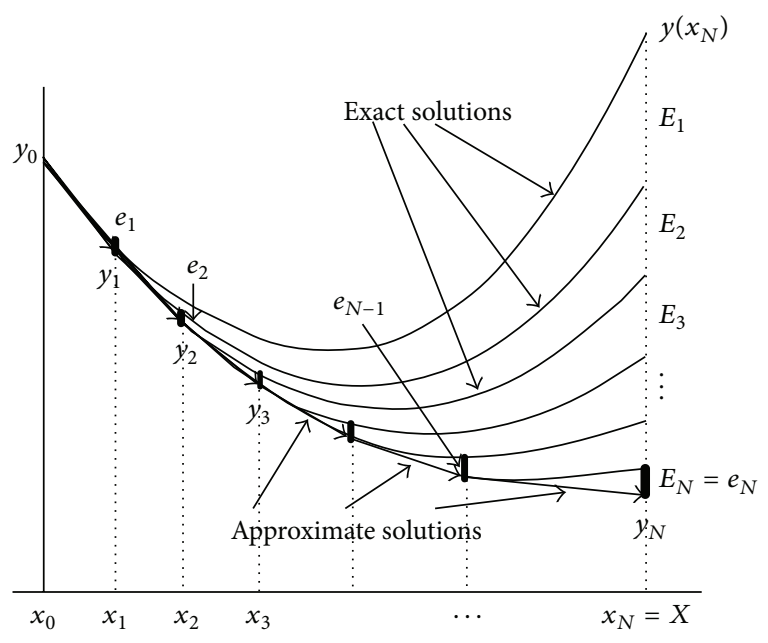

FIGURE 1: Global error estimation [42]. obtain

Estimate the local error using Theorem 5 and Figure 1 to

$$
\left\|e_{s}\right\| \leq C h_{s-1}^{(N+1) \beta} \text {. }
$$

From Theorem 7 with $\varepsilon=0$, we obtain

$$
\left\|E_{s}\right\| \leq\left\|e_{s}\right\| \exp \left(l\left(\frac{t_{N}^{\beta}}{\Gamma(\beta+1)}-\frac{t_{s}^{\beta}}{\Gamma(\beta+1)}\right)\right),
$$

from Figure 1, $E=E_{1}+E_{2}+\cdots+E_{N-1}+E_{N}$,

$$
\|E\| \leq \sum_{s=1}^{N}\left\|E_{s}\right\|
$$

as $h=\max _{1<s \leq N} h_{s-1}$, then we can deduce that

$$
h_{s-1}^{(N+1) \beta} \leq h^{N \beta} h_{s-1}^{\beta}
$$

and from (81)-(83), we deduce that

$$
\begin{gathered}
\|E\| \leq C h^{N \beta} \sum_{s=1}^{N} h_{s-1}^{\beta} \exp \left(l\left(\frac{t_{N}^{\beta}}{\Gamma(\beta+1)}-\frac{t_{s}^{\beta}}{\Gamma(\beta+1)}\right)\right) \\
=C h^{N \beta} I, \\
I \leq\left\{\begin{array}{l}
\int_{t_{0}}^{t_{N}} \exp \left(l\left(\frac{t_{N}^{\beta}}{\Gamma(\beta+1)}-\frac{t^{\beta}}{\Gamma(\beta+1)}\right)\right) d t \\
\int_{t_{0}}^{t_{N}} \exp \left(l\left(\frac{t_{N}^{\beta}}{\Gamma(\beta+1)}-h^{\beta}-\frac{t^{\beta}}{\Gamma(\beta+1)}\right)\right) d t
\end{array}\right.
\end{gathered}
$$


Then

$$
I \leq\left\{\begin{array}{l}
\frac{\Gamma(\beta)}{l t_{N}^{\beta-1}}\left(\frac{t_{N}}{t_{0}}\right)^{1-\beta} \\
\cdot \exp \left(l\left(\frac{t_{N}^{\beta}}{\Gamma(\beta+1)}-\frac{t_{0}^{\beta}}{\Gamma(\beta+1)}\right)-1\right), \\
\frac{\Gamma(\beta)}{l t_{N}^{\beta-1} \exp \left(-l h^{\beta}\right)\left(\frac{t_{N}}{t_{0}}\right)^{1-\beta} \quad \text { for } l \geq 0 ;} \\
\cdot \exp \left(l\left(\frac{t_{N}^{\beta}}{\Gamma(\beta+1)}-\frac{t_{0}^{\beta}}{\Gamma(\beta+1)}\right)-1\right),
\end{array}\right.
$$

A combination of (84)-(85) yields the desired result (77).

Remark 9 (see [43]). Since the global truncating error $E$ (77) tends to zero as $h$ tends to zero, then the proposed method (43) is convergent; that is, $|E| \rightarrow 0$ as $h \rightarrow 0$.

\section{Applications and Numerical Simulation}

In this section, we illustrate the theoretical results obtained in the previous sections. In order to do this we will consider the following examples whose exact solutions are known.

Example 1. Consider

$$
\begin{aligned}
& D_{t}^{(\beta)} y(t)= \frac{2}{\Gamma(3-\beta)} t^{2-\beta}-\frac{1}{\Gamma(2-\beta)} t^{1-\beta} \\
&+2 \tau t-\tau^{2}-\tau-y(t)+y(t-\tau), \\
& y(t)=t^{2}-t, \quad t \in[-\tau, 0] .
\end{aligned}
$$

The exact solution for this problem is $y(t)=t^{2}-t$.

For FDDEs (86) at $(\beta=1, \tau=1, h=0.25)$ and ( $\beta=0.1, \tau=0.1, h=0.25)$ the absolute errors between the computed points and exact ones are presented in Figures 2 and 3 respectively.

Example 2. Consider

$$
\begin{gathered}
D_{t}^{(3 / 4)} y(t)=y(t-1)-y(t)+g(t), \quad t>0, \\
y(t)=\sin (t), \quad t \in[-1,0],
\end{gathered}
$$

where $g(t)$ is defined by

$$
g(t)=\sin (t)-\sin (t-1)+4 \frac{t^{1 / 4}}{\Gamma(1 / 4)}{ }_{1} F_{2}\left[1 ; \frac{5}{8}, \frac{9}{8} ; \frac{-t^{2}}{4}\right]
$$

such that ${ }_{1} F_{2}$ is a special case of hypergeometric function.

The exact solution for this problem is $y(t)=\sin (t)$.

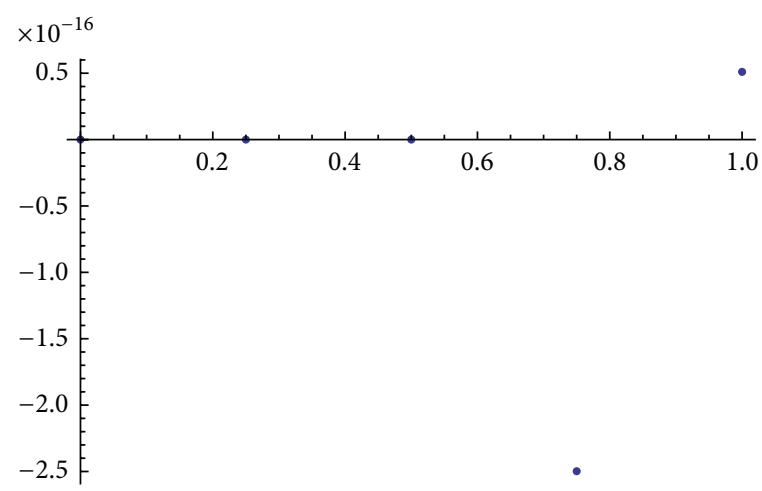

Figure 2: Plot of the absolute error with $h=0.25, t \in[0,1], \beta=1$, $\tau=1$.

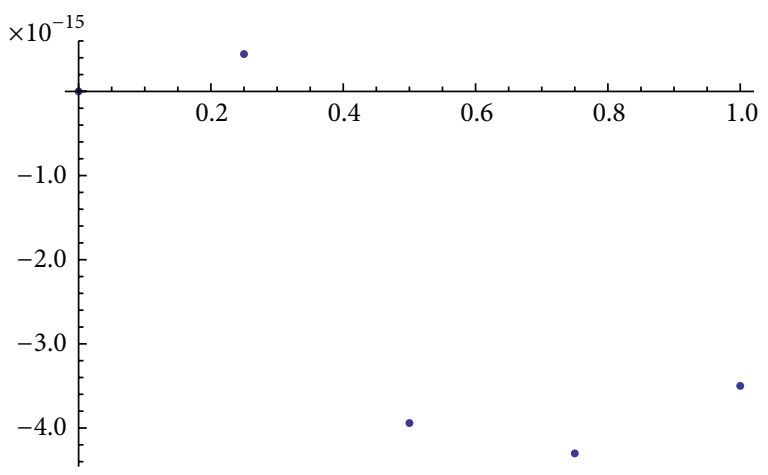

Figure 3: Plot of the absolute error with $h=0.25, t \in[0,1], \beta=0.1$, $\tau=0.1 \exp (-10 t)$.

In Table $3, h=1 / 2^{n} 10$, we present the results obtained with the proposed method at $N=4$ for different step sizes such that the maximum of the absolute error is given by

$$
\text { Error }=\max _{j}\left|y_{\text {exact }}\left(t_{j}\right)-y_{\text {numerical }}\left(t_{j}\right)\right|
$$

In the third column, the numerical convergence order which is defined by $\log _{2}(\operatorname{Error}(2 h) / \operatorname{Error}(h))$ is introduced.

Example 3. Consider

$$
\begin{gathered}
D_{t}^{(1 / 2)} y(t)=y(t-1)-y(t)+g(t), \quad t>0, \\
y(t)=\sin (t), \quad t \in[-1,0],
\end{gathered}
$$

where $g(t)$ is defined by

$$
g(t)=\sin (t)-\sin (t-1)+2 \sqrt{\frac{t}{\pi}}{ }_{1} F_{2}\left[1 ; \frac{3}{4}, \frac{5}{4} ; \frac{-t^{2}}{4}\right] .
$$

The exact solution for this problem is $y(t)=\sin (t)$.

In Table 4, the computational results of the maximum of absolute errors are displayed at $N=8$ for different time steps $h=1 / 2^{n} 10$ with $n=0,1,2,3,4,5$. In the third column, the numerical convergence order is introduced also. These results are in concordance with our theoretical results. 
TABLE 3

\begin{tabular}{lcc}
\hline$n$ & Error $(h)$ & Numerical convergence order \\
\hline 0 & 0.001761 & 2.73779 \\
1 & 0.000264 & 2.82329 \\
2 & 0.0000373 & 2.95177 \\
3 & $4.821 \times 10^{-6}$ & 3.07222 \\
4 & $5.732 \times 10^{-7}$ & 3.06486 \\
5 & $6.850 \times 10^{-8}$ & \\
\hline
\end{tabular}

TABLE 4

\begin{tabular}{ccc}
\hline$n$ & Error $(h)$ & Numerical convergence order \\
\hline 0 & $9.876 \times 10^{-6}$ & \\
1 & $7.876 \times 10^{-7}$ & 3.64839 \\
2 & $5.866 \times 10^{-8}$ & 3.74701 \\
3 & $3.856 \times 10^{-9}$ & 3.9272 \\
4 & $1.846 \times 10^{-10}$ & 4.38463 \\
5 & $8.360 \times 10^{-12}$ & 4.46476 \\
\hline
\end{tabular}

\section{Conclusion and Remarks}

In this paper, a new fractional order Runge-Kutta method based on BDF-type Chebyshev approximations is introduced. This approach is applicable to initial value problems for arbitrary order fractional differential equations with delay. This new method can be expressed as one-step recurrence formula. It is shown that the method may be formulated in an equivalent way as a Runge-Kutta method of order $N \beta$. The local and global truncating errors for this new scheme are obtained and proved. Numerical examples with constant delay or time varying delay are proposed to justify the effectiveness of the proposed scheme and find a good agreement with the theoretical results. For the future work, the method is also applicable to systems of fractional functional differential equations with delay.

\section{Conflict of Interests}

The authors declare that there is no conflict of interests regarding the publication of this paper.

\section{Acknowledgments}

This work was supported by Act 211 Government of the Russian Federation Program 02.A03.21.0006 on 27.08.2013 and by RFBR Grant 13-01-00089.

\section{References}

[1] R. Almeida and A. B. Malinowska, "A fractional calculus of variations for multiple integral with application to vibrating string," Journal of Mathematical Physics, vol. 51, pp. 1-12, 2010.

[2] P. J. Torvik and R. L. Bagley, "On the appearance of the fractional derivative in the behavior of real materials," Journal of Applied Mechanics, vol. 51, no. 2, pp. 294-298, 1984.
[3] A. Kilbas, H. Srivastava, and J. Trujillo, Theory and Applications of Fractional Differential Equations, North-Holland Mathematics Studies, 2006.

[4] J. Sabatier, O. P. Agrwal, and J. A. T. Machado, Advances in Fractional Calculus, Springer, 2007.

[5] E. H. Doha, A. H. Bhrawy, and S. S. Ezz-Eldien, "Efficient Chebyshev spectral methods for solving multi-term fractional orders differential equations," Applied Mathematical Modelling, vol. 35, no. 12, pp. 5662-5672, 2011.

[6] M. Inc, "The approximate and exact solutions of the spaceand time-fractional Burgers equations with initial conditions by variational iteration method," Journal of Mathematical Analysis and Applications, vol. 345, no. 1, pp. 476-484, 2008.

[7] H. Jafari and V. Daftardar-Gejji, "Solving linear and nonlinear fractional diffusion and wave equations by Adomian decomposition," Applied Mathematics and Computation, vol. 180, no. 2, pp. 488-497, 2006.

[8] M. M. Khader, "On the numerical solutions for the fractional diffusion equation," Communications in Nonlinear Science and Numerical Simulation, vol. 16, no. 6, pp. 2535-2542, 2011.

[9] M. M. Khader, T. S. El Danaf, and A. S. Hendy, "A computational matrix method for solving systems of high order fractional differential equations," Applied Mathematical Modelling, vol. 37, no. 6, pp. 4035-4050, 2013.

[10] M. M. Meerschaert and C. Tadjeran, "Finite difference approximations for fractional advection-dispersion flow equations," Journal of Computational and Applied Mathematics, vol. 172, no. 1, pp. 65-77, 2004.

[11] S. Y. Lukashchuk, "An approximate solution method for ordinary fractional differential equations with the RiemannLiouville fractional derivatives," Communications in Nonlinear Science and Numerical Simulation, vol. 19, no. 2, pp. 390-400, 2014.

[12] A. Bellen and M. Zennaro, Numerical Methods for Delay Differential Equations, Oxford Science Publications, Clarendon Press, Oxford, UK, 2003.

[13] R. Cooke and K. L. Bellman, Differential-Difference Equations, Academic Press, New York, NY, USA, 1963.

[14] R. Driver, Ordinary and Delay Differential Equations, Springer, Berlin, Germany, 1977.

[15] J. Hale, Theory of Functional Differential Equations, Springer, New York, NY, USA, 1977.

[16] V. G. Pimenov and E. E. Tashirova, "Numerical methods for solving a hereditary equation of hyperbolic type," Proceedings of the Steklov Institute of Mathematics, vol. 281, no. 1, pp. 126-136, 2013.

[17] M. Dehghan and R. Salehi, "Solution of a nonlinear time-delay model in biology via semi-analytical approaches," Computer Physics Communications, vol. 181, no. 7, pp. 1255-1265, 2010.

[18] N. MacDonald, Biological Delay Systems: Linear Stability Theory, Cambridge University Press, 1989.

[19] S. Bhalekar, "Dynamical analysis of fractional order Uçar prototype delayed system Sachin Bhalekar," Signal, Image and Video Processing, vol. 6, no. 3, pp. 513-519, 2012.

[20] S. Bhalekar, V. Daftardar-Gejji, D. Baleanu, and R. Magin, "Fractional Bloch equation with delay," Computers and Mathematics with Applications, vol. 61, no. 5, pp. 1355-1365, 2011.

[21] R. L. Magin, "Fractional calculus models of complex dynamics in biological tissues," Computers and Mathematics with Applications, vol. 59, no. 5, pp. 1586-1593, 2010. 
[22] A. Si-Ammour, S. Djennoune, and M. Bettayeb, "A sliding mode control for linear fractional systems with input and state delays," Communications in Nonlinear Science and Numerical Simulation, vol. 14, no. 5, pp. 2310-2318, 2009.

[23] D. Wang and J. Yu, "Chaos in the fractional order logistic delay system," Journal of Electronic Science and Technology, vol. 6, no. 3, pp. 225-229, 2008.

[24] Z. Yang and J. Cao, "Initial value problems for arbitrary order fractional differential equations with delay," Communications in Nonlinear Science and Numerical Simulation, vol. 18, no. 11, pp. 2993-3005, 2013.

[25] F. Chen and Y. Zhou, "Attractivity of fractional functional differential equations," Computers and Mathematics with Applications, vol. 62, no. 3, pp. 1359-1369, 2011.

[26] V. Lakshmikantham, "Theory of fractional functional differential equations," Nonlinear Analysis, Theory, Methods and Applications, vol. 69, no. 10, pp. 3337-3343, 2008.

[27] K. Krol, "Asymptotic properties of fractional delay differential equations," Applied Mathematics and Computation, vol. 218, no. 5, pp. 1515-1532, 2011.

[28] S. Bhalekar and V. Daftardar-Gejji, "A predictor-corrector scheme for solving nonlinear delay differential equations of fractional order," Journal of Fractional Calculus and Applications, vol. 1, no. 5, pp. 1-9, 2011.

[29] Z. Wang, "A numerical method for delayed fractional-order differential equations," Journal of Applied Mathematics, vol. 2013, Article ID 256071, 7 pages, 2013.

[30] Z. Wang, X. Huang, and J. Zhou, "A numerical method for delayed fractional-order differential equations: based on G-L definition," Applied Mathematics and Information Sciences, vol. 7, no. 2, pp. 525-529, 2013.

[31] M. L. Morgado, N. J. Ford, and P. M. Lima, "Analysis and numerical methods for fractional differential equations with delay," Journal of Computational and Applied Mathematics, vol. 252, pp. 159-168, 2012.

[32] B. P. Moghaddam and Z. S. Mostaghim, "A novel matrix approach to fractional finite difference for solving models based on nonlinear fractional delay differential equations," Ain Shams Engineering Journal, vol. 5, no. 2, pp. 585-594, 2014.

[33] B. P. Moghaddam and Z. S. Mostaghim, "A numerical method based on finite difference for solving fractional delay differential equations," Journal of Taibah University for Science, vol. 7, no. 3, pp. 120-127, 2013.

[34] H. Ramos and J. Vigo-Aguiar, "A fourth-order Runge-Kutta method based on BDF-type Chebyshev approximations," Journal of Computational and Applied Mathematics, vol. 204, no. 1, pp. 124-136, 2007.

[35] K. Diethelm and N. J. Ford, "Analysis of fractional differential equations," Journal of Mathematical Analysis and Applications, vol. 265, no. 2, pp. 229-248, 2002.

[36] K. S. Miller and B. Ross, An Introduction to the Fractional Calculus and Fractional Differential Equations, John Wiley \& Sons, New York, NY, USA, 1993.

[37] I. Podlubny, Fractional Differential Equations, Academic Press, New York, NY, USA, 1999.

[38] S. Samko, A. Kilbas, and O. I. Marichev, Fractional Integrals and Derivatives: Theory and Applications, Gordon and Breach, Yverdon, The Netherlands, 1993.

[39] D. Funaro, Polynomial Approximation of Differential Equations, Springer, New York, NY, USA, 1992.
[40] C. W. Clenshaw and A. R. Curtis, "A method for numerical integration on an automatic computer," Numerische Mathematik, vol. 2, no. 1, pp. 197-205, 1960.

[41] J. C. Butcher, Numerical Methods for Ordinary Differential Equations, John Wiley \& Sons, New York, NY, USA, 2nd edition, 2008.

[42] E. Hairer, S. P. Nrsett, and G. Wanner, Solving Ordinary Differential Equations I Nonstiff Problems, Springer, Berlin, Germany, 2008.

[43] R. J. Le Veque, Finite Difference Methods for Differential Equations, University of Washington, 2005. 


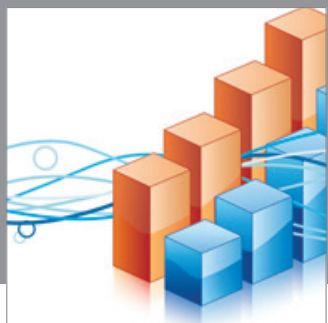

Advances in

Operations Research

mansans

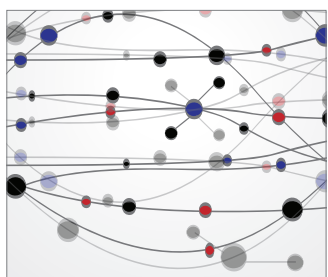

The Scientific World Journal
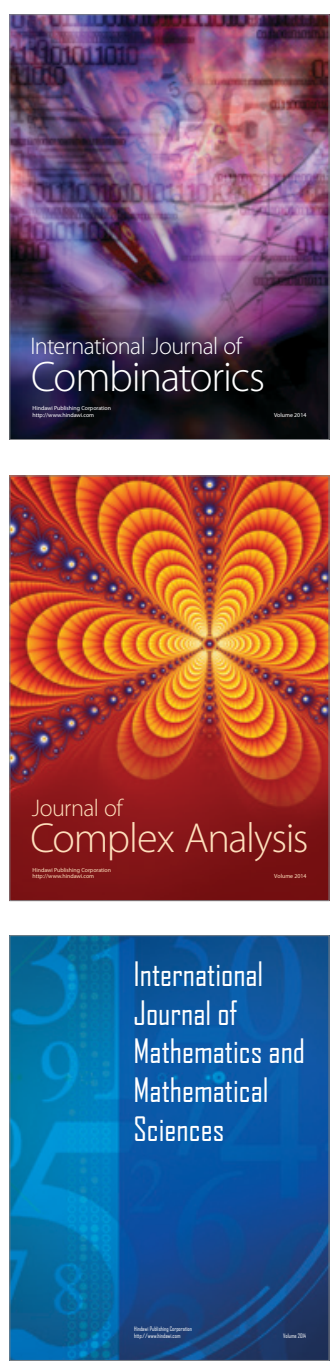
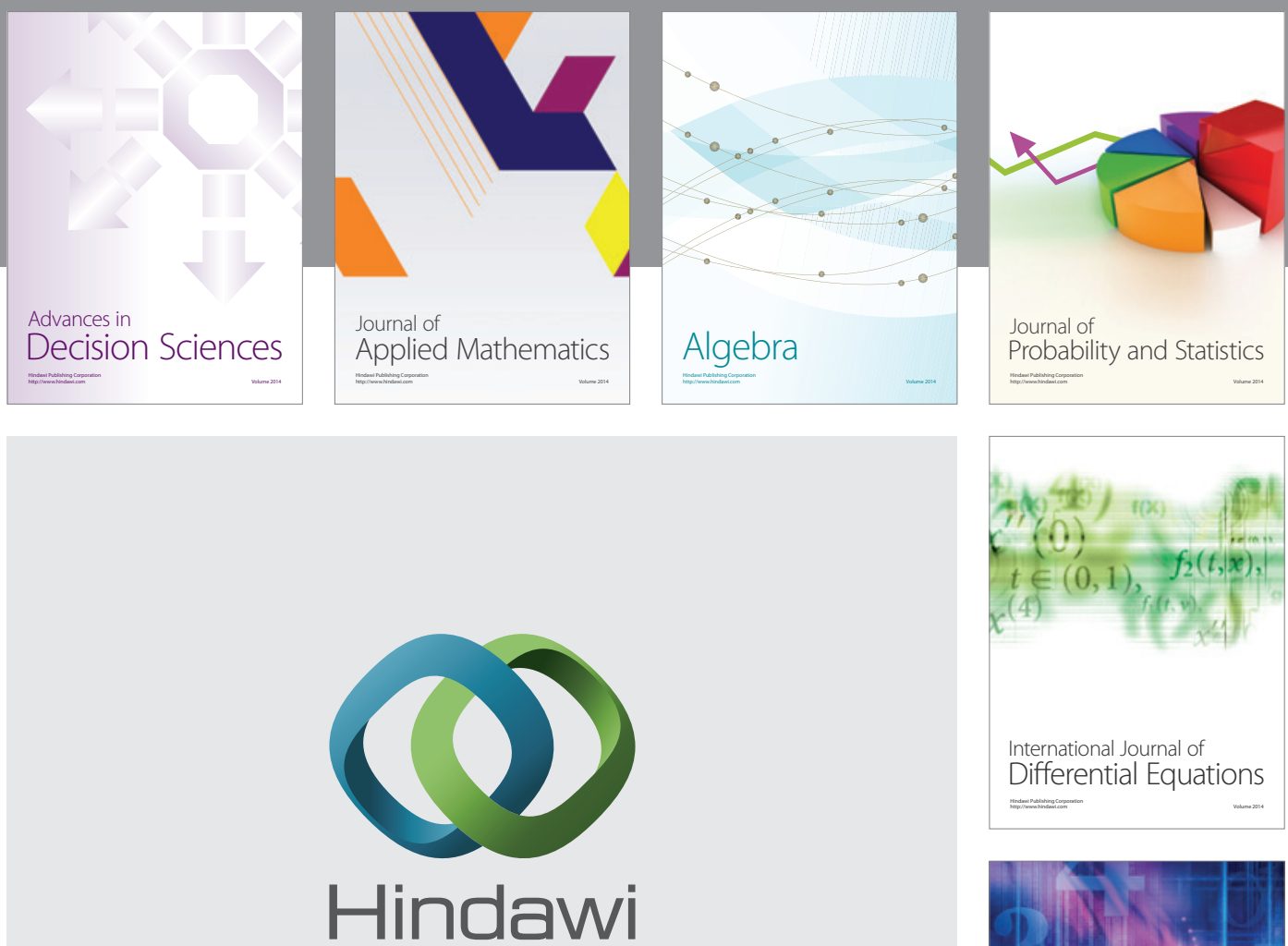

Submit your manuscripts at http://www.hindawi.com
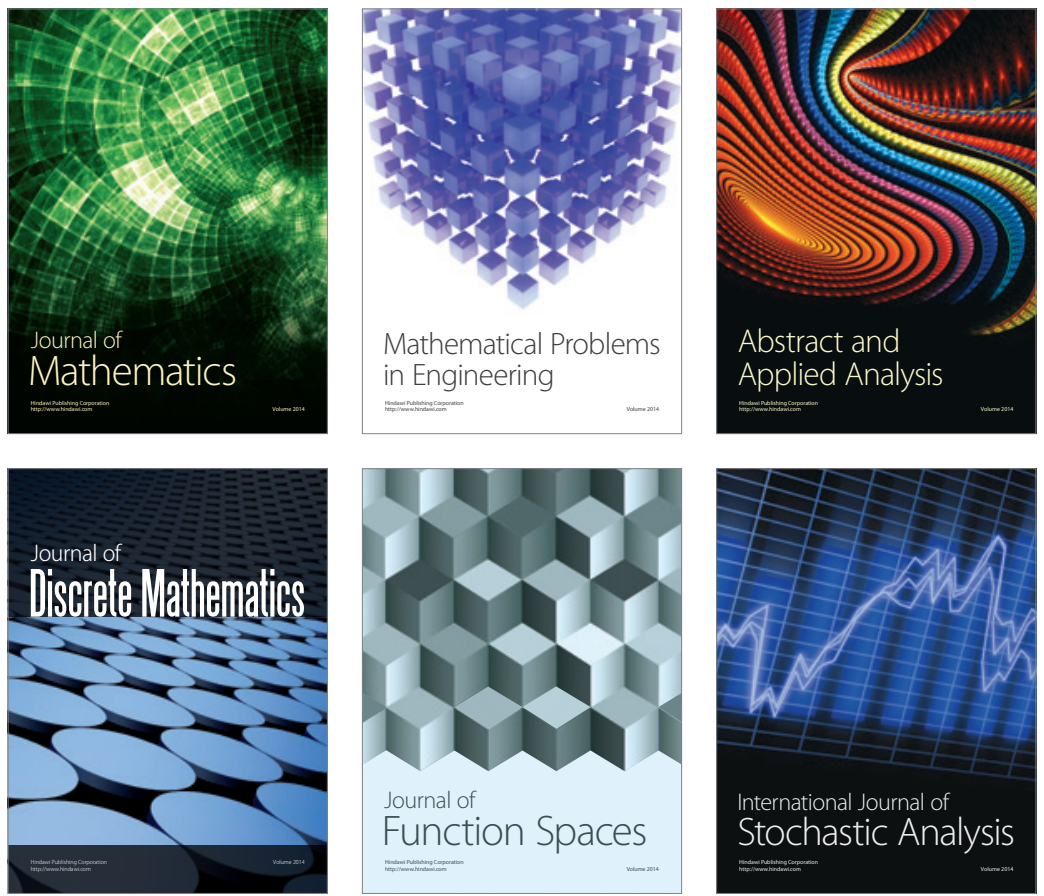

Journal of

Function Spaces

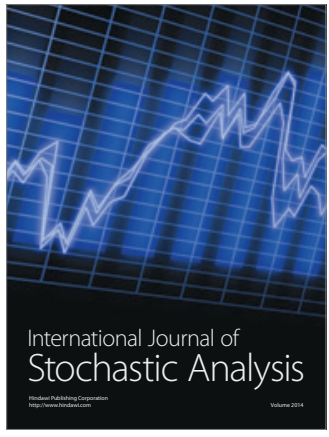

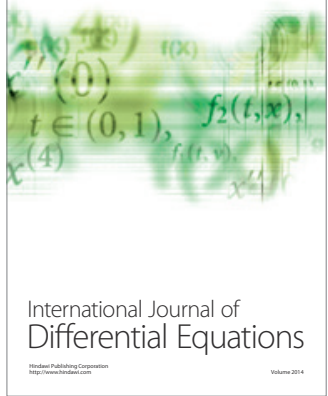
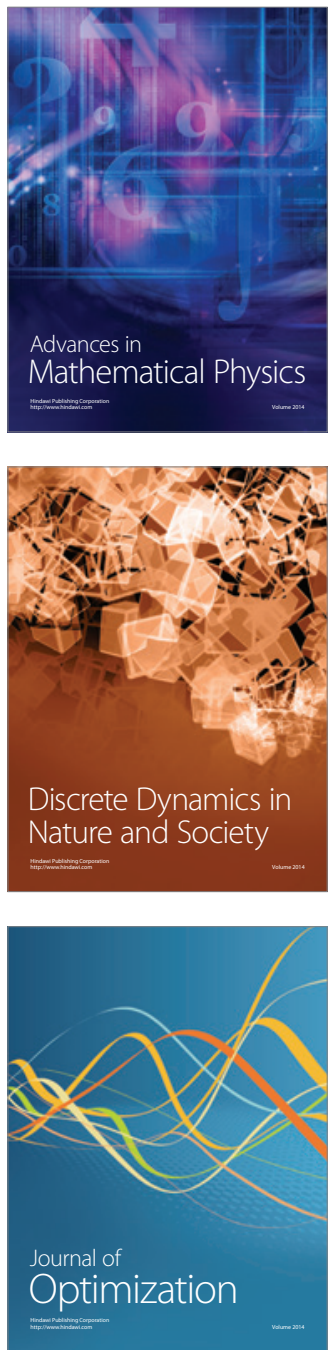